\title{
Philosophiques
}

\section{Chantal Saint-Jarre, Du Sida, l'Anticipation imaginaire de la mort et sa mise en discours, Paris, Denoël, 1994, 267 p.}

\section{Isabelle Lasvergnas}

Volume 23, numéro 2, automne 1996

URI : https://id.erudit.org/iderudit/027416ar

DOI : https://doi.org/10.7202/027416ar

Aller au sommaire du numéro

Éditeur(s)

Société de philosophie du Québec

ISSN

0316-2923 (imprimé)

1492-1391 (numérique)

Découvrir la revue

Citer ce compte rendu

Lasvergnas, I. (1996). Compte rendu de [Chantal Saint-Jarre, Du Sida, l'Anticipation imaginaire de la mort et sa mise en discours, Paris, Denoël, 1994, 267 p.] Philosophiques, 23(2), 456-461. https://doi.org/10.7202/027416ar d'utilisation que vous pouvez consulter en ligne.

https://apropos.erudit.org/fr/usagers/politique-dutilisation/ 
Chantal Saint-Jarre, Du Sida, l'Anticipation imaginaire de la mort et sa mise en discours, Paris, Denoël, 1994, 267 p.

Ce texte reprend les propos d'une communication présentée dans le cadre d'une table ronde sur le liure de Chantal Saint-Jarre, organisée par la Société de philosophie du Québec à l'occasion du Congrès de l'ACFAS qui s'est tenu à Chicoutimi (23 mai 1995). 
Ecrit-on jamais, autrement, qu' in memoriam " ? Sans doute que non. Et certainement pas lorsque la matière première d'un ouvrage provient d'une expérience clinique de l'auteur. Certains textes, toutefois, revendiquent plus que d'autres ce statut de naissance, ce destin, et c'est bien là le cas du livre de Chantal Saint-Jarre. Du Sida, l'Anticipation imaginaire de la mort et sa mise en discours (1994), livre qui a valu à son auteur le Prix du Gouverneur général du Canada la même année.

Dans cette œuvre cri. dans ce témoignage sans nul doute à visée politique et militante. l'auteur parle publiquement au nom des sidéens qui sont déjà disparus et contribue à projeter leurs voix sur la scène publique. Mais il s'agit aussi d'un témoignage singulier et c'est par cette part de singularité, assumée, souffrante, que le travail de Chantal Saint-Jarre nous rejoint de façon plus sensible.

Il est habituel aux psychanalystes de souligner que des mouvements de contre-transfert innervent les actes d'écriture et les enonciations théoriques. Nul doute qu'à propos de cet ouvrage nous pourrions parler à tout le moins, dans le ton de son écriture, de " contre-émotionnalité " nettement perceptible et, répondant en écho à l'intensité des mouvements psychiques internes, à l'œuvre chez les personnes séropositives ou sidéennes, rencontrées. Une trame à peine retenue de douleur, de passion et de deuil imprime le ton général de l'ensemble du texte.

Car ce livre est en effet un reste. Reste à l'intérieur de soi, pour Chantal Saint-Jarre de ses rencontres, dans des groupes de soutien ou de son travail en psychothèrapie, avec des malades assaillis d'angoisse, de terreur et parfois de haine, devant l'immanence de leur mourance réelle. Patients. dont on peut supposer de surcroit qu'ils sont aujourd'hui tous décédés, une des raisons parmi lesquelles l'auteure témoigne en leur nom. Auteure qui se place en position de survivante, après avoir été en état de veille, et qui est peut-être encore, aussi. en état de pleureuse au moment oủ elle écrit.

En matière de "restes ", on peut déceler dans le travail de Chantal SaintJarre des traces d'émotions, mais également des traces d'enjeux pluriels par rapport auxquels un thérapeute se trouve interpellé - peut-ètre méme écartelé - lorsque sa pratique de travail l'amène à intervenir dans un contexte où la mort qui va détruire le corps et le sujet est déjà intensément a l'ouvre.

Comment se positionner psychothérapiquement parlant lorsque ce dont il est question, c'est du réel, et ceci dans un proche avenir, et non pas "seulement" d'un phantasme de mort qui serait agi par l'angoisse de castration ? Comment ètre "soignant "lorsqu'on est déjà engagé avec son patient dans "une Chronique de la mort annoncee "? Comment s'inscrire éthiquement comme sujet politique, lorsqu'on entend ètre le porte-parole de ceux qui sont en train de perdre la parole ou de ceux qui l'ont déjà perdue ? Position subjective qu'il est difficile de faire émerger et de clarifier et dont la difficulté ne s'estompe pas pour autant lorsqu'on choisit de se faire le porte-voix de cette part de la douleur humaine. De cette part de la tragédie humaine.

Le témoignage que choisit de faire Chantal Saint-Jarre participe sans nul doute du processus de deuil omniprésent auquel elle a été assignée en tant que thèrapeute dont la pratique clinique s'inscrit sous les auspices de la mort incessante. Comment maintenir en soi mème un mouvement vivant de psychisation quand on est assailli par un environnement de mort et par l'impasse qu'imprime en termes de temps disponible dans la relation therrapeutique, le diagnostic fatal dont est marqué chacun des patients que l'on rencontrera ? Posture de désespérance curative a priori, posture d'impuissance où la neutralité de l'être ne peut se soutenir. 
C'est pourquoi à plusieurs égards dans sa forme d'écriture, dans sa construction, le propos développé parait parfois osciller entre effets du traumatisme sur la capacité de mise en pensée et tentative de resymbolisation.

On ne peut se surprendre tout à fait de cette oscillation entre deux pōles. La question de la mort immanente, réelle, saisit le psychanalyste à de multiples niveaux. Elle le saisit aussi. dans une certaine indécidabilité au lieu de son éthique et de sa technique. dans sa potentialité d'aide "réelle dans les réferents théoriques qui sous-tendent les modalités de son écoute et de ses interventions. Comment intervenir? Privilegier la réalité du traumatisme, la reconnaitre et s'y tenir au plus près? Ou bien, privilégier l'écoute des fantasmes qui ont été réactivés à cette occasion, et qui signeraient le plus intime du sujet dans son bagage imaginaire? Filiation théorico-clinique, plutôt dans le sillage de Ferenczi, ou plutôt dans celui de Freud pour aller vite, mème si Ferenczi n'a pas été explicitement nommé dans le texte.

Afin de se dégager quelque peu de l'effet accablant, vidant, anxiogène. traumatique en fin de compte, que ne peut manquer de constituer à terme, pour le thérapeute lui-mème, la répétition des morts qu'il aura à vivre, le recours dans l'après-coup à l'écriture, constitue peut-être une des meilleures voies possibles.

Comment, en effet, assimiler ces maternitès psychothérapeutiques dont la destinée est d'avance déjà signée: celle d'être avortées ? Comment leur survivre ? Douleur de l'enfant mort qui étreint le thérapeute, le touche en plein cœur, l'envahit, lui, et pas seulement son patient. C'est pourquoi personnellement je ne me contenterais pas de la lecture qui nous est proposée par rapport à cette figure de "l'enfant-mort ", et qui constitue l'axe organisateur de l'analyse. "Douleur de l'enfant mort " attribuée, me semble-t-il, de façon trop explicite au(x) patient(s) d'abord et, en particulier, au cas de "Joseph " dont l'auteure a été bouleversée, habitée, lestée, au point d'en devoir écrire son livre touchant.

De même, on peut se demander si au fil du développement argumentatif, les diverses réfërences emblématiques empruntées à l'histoire de l'art, ne recouvrent pas quelque part des formes de mouvements identificatoires du thérapeute à l'endroit de ses patients.

On se souvient que les figures évoquées dans le livre sont toutes inscrites dans des formes de gemellités nourricières contradictoires, mortifères d'un côté et secourables, nourrissantes, de l'autre: le jumeau mort dont on serait l'incarnation nécronymique ou, le frère-mère ou la sœur-mère - Thêo pour Van Gogh. Galia, pour Dali - gemellités inscrites entre le poison intime qui gruge silencieusement de l'interieur et ce que Ferenczi nommait le "bandage psychique " à savoir, ce qui permet, vaille que vaille, de tenir, mème si c'est bien précairement, à la vie.

Dans le contenu manifeste du texte il apparait au lecteur que, du point de vue psychothérapique, c'est à partir de la position de personne secourable, dans sa fonction predominante de " bandage psychique * que Chantal Saint-Jarre espère pouvoir intervenir lors de ces ètats extrêmes de "commotion qui modifient le soi "(S. Ferenczi, Joumal clinique, p. 249). En particulier avec son patient Joseph, quand "l'amour et la force de l'analyste, en supposant que sa confiance en lui aille suffisamment profond et soit suffisamment grande. agissent à peu près comme l'étreinte d'une mère aimante et d'un père protecteur * (Ibid., p. 120).

Soutenir, contenir, afin que ce qui risquerait de devenir * une temporalité morte * puisse ètre une temporalité encore "vive *. 
Au plan contre-transférentiel toutefois, dans quelle mesure une part d'identification à l'être du patient gagné par l'infiltrat mortel n'est-elle pas silencieusement en jeu chez le thérapeute? Et ceci, d'autant plus qu'elle n'a pas pu ne pas être assignèe, dans certaines projections transférentielles à son endroit, à ẽtre, elle aussi, vécue comme ètant "mortifère ". Ainsi que l'a èté pour le patient, l'infiltrat contaminateur venu de l'extérieur et, dans le cas des hèmophiles, injecté " de force ", à l'intérieur d'eux-mèmes.

Le livre de Chantal Saint-Jarre contient toutefois une visée autre que le seul développement d'une réflexion clinique. Celle-ci, en effet, aux yeux de l'auteure. se doit d'ètre mise au service d'une pensée collective renouvelée concernant le statut social attribué aux malades atteints du sida. Si lon se situe dans une filiation foucaldienne, on peut dire que le sida convoque chacun d'entre nous à révéler ce qu'il est à titre de sujet politique.

Le sida réveille les grandes peurs moyenāgeuses. Cette pandémie qui mêle le sexe, le sang, la mort gagne du terrain et multiplie ses victimes. Elle ne touche plus seulement les homosexuels, les "junkies", mais aussi les hémophiles, les femmes infestées par des partenaires n'ayant pas respecté les règles du "safer-sex", les bèbés contaminés dans le sein de leur mère. Les " coupables "par rapport aux "innocents". Les victimes transformées en assassins (cf. Stephan Shecter, "Considérations post-modernes sur le SIDA ", notes de recherche, Anthropologie et Sociétés, $n^{\text {os }} 2-3,1991$, p. 105-114). Car contrairement à la tuberculose, à la "bactérie mangeuse de chair ", à la maladie Ébola, dans la terreur rampante que fait naitre le sida, se niche lillusion qu'un groupe humain peut ètre désigné comme porteur du fléau. comme agent initialement transmetteur, comme responsable.

Serait-ce cette assignation politique de l'autre au lieu de la culpabilité qui pourrait aider à comprendre la tendance récente à la recrudescence de la maladie observée dans les pays occidentaux ? "La frilosité des responsables à la communication à communiquer sur la sexualité [...] ne suffit pas à expliquer le phénomène. Les fantasmes, le désir et donc la dimension inconsciente [...] semble davantage déterminer les changements de comportement $[\ldots]$ une minorité d'homosexuels allant jusqu'à revendiquer une séroconversion volontaire ", relatait-on récemment dans le journal Le Monde (14-15 mai 1995).

Se pourrait-il donc que, au plan imaginaire, dans une nouvelle forme d'équivalence des significations, dans un effet mètonymique, ètre homosexuel aujourd'hui équivaille plus ou moins à être porteur du V.I.H. ? Les "gais " seraient-ils insidieusement aspirés, à leur insu, dans une identification nécronymique d'une autre sorte que celle précédemment évoquée dans le propos du livre?

Dans un témoignage rapporté par Le Monde (14-15 mai 1995), un témoin, "Laurent ", parle de sa tentation suicidaire - la séroconversion - dans des termes qui font penser à un nouveau romantisme : "ètre séropositif pour être comme les autres sans doute, peut-être par culpabilisation vis-à-vis des disparus, vis-à-vis des amis séropos encore en vie [...]. Cette putain de logique du ghetto encore". Dans ses propos, toutefois, "Laurent "évoque davantage que le seul holocauste de soi-même mù par de la culpabilité. Il nomme une tentative folle, "débile", selon ses termes, de conserver par ce pacte de sang entre "frères-maudits", quelque chose d'une radicalité du groupe dont l'aphorisme ironique " gay is beautiful " avait voulu autrefois politiquement témoigner

Étre solidaire, ètre infecté, ètre porteur, ètre coupable : chaîne de contiguité sémantique infernale qui emprisonne le sujet dans un destin de paria, destin privé sans doute, mais destin socio-politique également, qui l'assimile à certains précédents historiques dont la mémoire n'est jamais totalement effacée. Au XV 
siècle, on s'en souvient, lors de la Grande Peste, les juifs avaient été soupçonnés, puis accusés, d'être les déclencheurs du Mal.

Max Weber rappelle qu'un peuple paria est un peuple höte vivant dans un environnement dont il est l'êtranger et par rapport auquel il doit ètre maintenu en état de séparation par des mécanismes rituels. À cela. Hannah Arendt ajoute qu'il faut faire de la catégorie paria, une catégorie non pas subjective, mais politique. Or le sida vient révéler l'extrème fragilisation des difficiles acquis de ce que fut le mouvement gay. Mouvement critique, - contre-culturel, comme on disait dans les années soixante-dix - et dont les pratiques, en particulier celle d'émancipation sexuelle - jusqu'au S.M. - venaient re-solliciter et exacerber chez les "straights " les hantises les plus archaïques de la transgression, de la violence et de la dangerosité qui seraient inhérentes à l'état de déliaison du sexuel.

À cet égard, il me semble qu'il faut regretter, en particulier, une forme de lacune importante dans l'analyse de Chantal Saint-Jarre. Cette lacune touche à l'inscription symbolique conférée dans le social, d'une part, aux hémophiles contaminés par un système gestionnaire de transfusion sanguine, scandaleusement défaillant et, d'autre part, aux homosexuels contaminés par leur propre pratique sexuelle. Bien entendu, Chantal Saint-Jarre appelle avec vigueur à la compassion collective indifférenciée à l'endroit de tous les malades victimes du sida. Mais la compassion, ce n'est pas assez. Car le fait de se contenter d'exalter. mème si c'est plus qu'à juste titre. l'horreur et l'injustice de la situation faite aux hémophiles, tandis que l'on tait la part du sexuel comme cause manifeste de la maladie chez les homosexuels, rejoint la séparation maintenue dans l'opinion collective entre ceux qui seraient des victimes de la maladie et ceux qui en seraient des agents propagateurs. Trichotomie entre ètre inoculé, s'auto-inoculer et inoculer l'autre, dans laquelle se logerait la coupure entre la victime et l'assassin.

Je dirais qu'il est facile d'un point de vue d'une morale "ordinaire " de dénoncer le fléau d'un système de gestion des soins bureaucratique et anomique qui sacrifie, dans l'indifférence des innocents. Plus, qui transforme en victimes ceux qui devraient ètre les bénéficiaires des services offerts. Assassinat collectif à bas bruit, génocide anonyme, de la part d'administrateurs qui, à l'instar du teneur de livre du Château de Kafka, ne sont plus des personnes à part entière, c'est-à-dire capables de jugement autonome et singulier, mais les vecteurs soumis d'un système ; opérateurs sans état d'âme qui n'ont plus pour seul génie que celui d'appliquer avec zèle des normes d'exécution. Normes, dont ils deviennent les robots au lieu d'en ètre les critiques potentiels et les interprètes.

Goretta/Eichman, association souvent suggérée. Assimilation tentante, mais au prix de quelle simplification de l'analyse? Au prix de quel aveuglement et de quelle impuissance du collectif? Dans quelle mesure Goretta, tout comme l'avait fait Eichman avant lui, en renvoyant en dehors de lui-même, en dehors de sa capacité propre de jugement, à des instances supérieures la responsabilité des actes perpétrès, ne révèle-t-il pas à son tour que c'est non seulement le sujet contemporain qui a perdu tout refërent d'exigence éthique, mais également le politique, qui en tant que tel a disparu, s'est dissous, pour n'ètre plus que du gestionnaire. du bureaucratique, du calcul informatisé et financier. De même, à travers la personne de Goretta, en cédant si volontiers aux clameurs sanguinaires qui accompagnent les jeux du cirque, en sacrifiant à la vax populi un bouc émissaire, l'instance gourvernementale ou ses tenants-lieu qui prêtendent encore représenter le politique, ne se dédouane-t-elle pas à bon compte d'une responsabilité qu'elle n'est mème plus en mesure de se représenter à elle-même? 
En revanche, en assimilant d'avance les sidéens homosexuels à des pratiques de vie soi-disant "déviantes ", en en faisant les responsables du mal mortel dont ils sont atteints, la société ne les rejette-t-elle pas à la seule solidarité où ils puissent se sentir encore admis, celle de leur "groupe d'homos " dans une appartenance commune à l'impur, à la culpabilité, au véhicule de transmission de la contamination. Et ne les condamne-t-elle pas insidieusement, froidement, au devoir de disparaitre ? De se faire disparaitre ? Leur exclusion serait-elle la condition pour que le reste de la société conserve illusoirement l'impression de sa sauvegarde, de son innocence, et de son asepsie ?

Le sexe, le sang, la mort, pourquoi cette chaine associative transporte-t-elle une telle potentialité de surchauffe sémantique ? À quelle primitivité en chacun de nous, "le sang impur ", indissociablement relié au sexe et au tabou de la virginite, renvoie-t-il ? Doit-on encore rappeler avec Freud que "ce n'est pas seulement le premier coï avec la femme qui est tabou : tous les rapports sexuels le sont [...] [car] là où le primitif a posé un tabou, c'est qu'il redoute un danger et on ne peut rejeter le fait que toutes ces prescriptions d'évitement trahissent une crainte essentielle " ( Le tabou de la virginité ". p. 71).

Ainsi, en dépit de, et par-delà l'indiscutable nécessité de précaution prophylactique qu'il représente, dans quelle mesure l'encouragement au recours au condom promu par les politiques préventives en santé publique ne constitue$\mathrm{t}$-il pas aussi quelque part, une manifestation, dans la langue du banal, de ce qui, au plan inconscient, se maintient toujours au niveau de la phobie du sexuel ? Alors mème, qu'en termes de représentation dans plusieurs des formes de ce qui apparait bien ètre une terreur collective, la métonymie sida/homosexualité semble occuper une place prépondérante et prêter figure à un nouveau spectre de l'angoisse de la mort et de l'angoisse du sexuel, indissociablement conjuguées, en cette fin du $\mathrm{XX}^{\mathrm{e}}$ siècle.

Isabelle Lasvergnas

Département de sociologie

Université du Québec à Montréal 